\title{
Shaken, not Stirred: the Ancestry of the Starburst Galaxy NGC 253
}

\author{
T. J. Davidge \\ Herzberg Institute of Astrophysics, \\ National Research Council of Canada, 5071 West Saanich Road, \\ Victoria, BC Canada V9E 2E7 \\ email: tim.davidge@nrc.ca
}

\begin{abstract}
Near-infrared images obtained with WIRCam are used to investigate the recent history of the starburst galaxy NGC 253. The distribution of stars in the disk is lopsided, with the projected density of young and intermediate age stars in the north east portion of the disk higher than on the opposite side of the galaxy. Bright AGB stars are also detected out to 15 kpc above the disk plane. Comparisons with models suggest that the extraplanar stars formed over a broad range of ages, suggesting that the disk of NGC 253 was disrupted by a tidal encounter.
\end{abstract}

Keywords. galaxies: individual (NGC 253) - galaxies: evolution - galaxies: spiral

\section{INTRODUCTION}

Nearby starburst galaxies provide glimpses into conditions that were common at high redshift. The nearby starburst galaxy NGC 253 is the largest member of the Sculptor group. The disk contains numerous filamentary and bubble-like structures that are signatures of a high SFR, indicating that the elevated levels of star-forming activity in NGC 253 have not been restricted to the central regions of the galaxy.

We have used the CFHT WIRCam to obtain deep near-infrared observations of three $20 \times 20$ arcmin fields, with the goal of mapping the distribution of the brightest red stars. The images sample the major axis of NGC 253 at both ends of the disk (Fields 1 and 2) and the south east segment of the minor axis (Field 3) with an angular resolution $\sim 0.9$ arcsec. The total exposure time per field is $2800 \mathrm{sec}$ in $J$ and $5600 \mathrm{sec}$ in $K s$.

Sources were sorted into disk and extraplanar components using distance from the principle axis of the galaxy. Stars that fall within a 120 arcsec wide strip along the major axis are assumed to be in the disk, while those outside of the disk strip are assumed to be extraplanar.

\section{THE DISK OF NGC 253}

Color-magnitude disgrams (CMDs) of stars near $\mathrm{R}_{G C}=10 \mathrm{kpc}$ along the disk plane are compared with solar metallicity isochrones from Girardi et al. (2002) in Figure 1. The peak brightness of the AGB concentration is consistent with an age $\sim 0.1$ Gyr, indicating that the disk of NGC 253 has been actively forming stars throughout the recent past. The $10 \mathrm{Myr}$ and $30 \mathrm{Myr}$ isochrones define a near-vertical RSG sequence near the bright end of the CMDs, and there is a rich RSG sequence in the Field 2 CMD.

Samples of M giants, C stars, and RSGs were extracted based on location in the $(K, J-K) \mathrm{CMD}$, and Figure 2 shows the spatial distribution of each stellar type. The concentration of RSGs in the north east part of the disk indicates that this area had a 


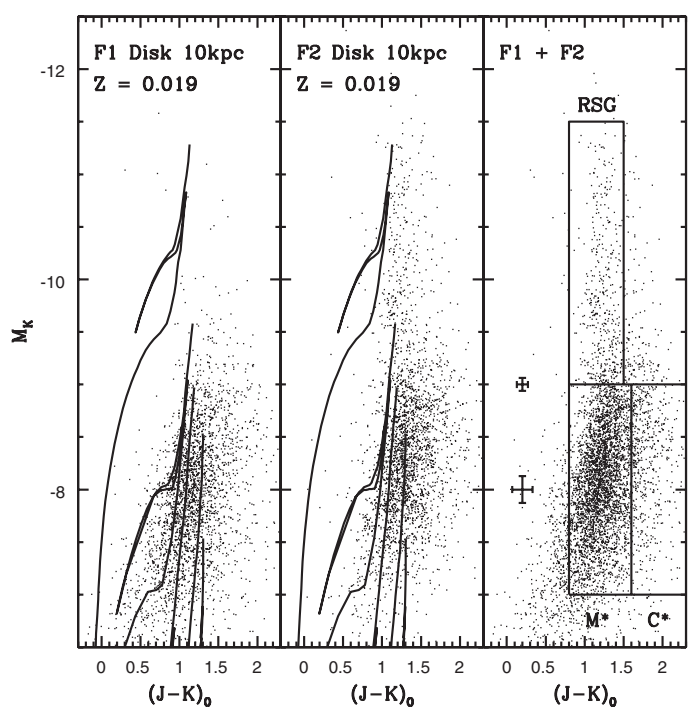

Figure 1. The $\mathrm{R}_{G C}=10 \mathrm{kpc}$ CMDs of disk stars are compared with solar metallicity isochrones from Girardi et al. (2002). The error bars in the right hand panel show $1 \sigma$ uncertainties in the photometric measurements as estimated from artificial star experiments. Isochrones with ages $10 \mathrm{Myr}, 30 \mathrm{Myr}, 100 \mathrm{Myr}, 1 \mathrm{Gyr}$, and $10 \mathrm{Gyr}$ are also shown. The photometry has been corrected only for foreground extinction, and the difference in $J-K$ between the isochrones and RSGs in Field 2 is probably due to extinction that is internal to NGC 253. The majority of the AGB stars detected with WIRCam in NGC 253 have ages of only a few Gyr. The areas that are used to define the M giant, C star, and RSG samples that are plotted in Figure 2 are indicated in the right hand panel, which shows a composite of the Field 1 and Field 2 CMDs.

higher SFR than elsewhere in the disk during the past few tens of Myr. Bright M giants can be traced to larger distances along the major axis than RSGs, suggesting that the most recent episodes of star formation have been confined to small and intermediate radii in the disk. The central 'hole' in the $\mathrm{M}$ giant distribution is due to crowding, which hinders the detection of individual stars. The distribution of $\mathrm{M}$ giants in the disk of NGC 253 is lopsided, in the sense that the density of $\mathrm{M}$ giants in the outer disk is higher in the north west quadrant than in the south east quadrant; this difference amounts to $\sim 0.5$ dex, and occurs at all radii. Structures that may be signatures of an interaction with a companion can be seen in the $\mathrm{M}$ giant distribution. One such structure is the spur in the $\mathrm{M}$ giant distribution that extends to the south east of the disk in Field 1, and this feature is also evident in the deep images discussed by Beck et al. (1982) and Malin \& Hadley (1997). This is an area of $\mathrm{H} \alpha$ emission, but there is no obvious UV emission, suggesting that there is not a large concentration of young stars (Hoopes et al. 2005).

\section{THE EXTRAPLANAR ENVIRONMENT}

A remarkable discovery is that the extraplanar regions of NGC 253 contain large numbers of stars that formed at least 1 Gyr in the past. The LFs of extraplanar stars have been compared with model LFs that were constructed from routines in Starfish (Harris \& Zaritsky 2001) for SSPs and a solar metallicity system that has experienced a constant SFR over the age range $0.2-10 \mathrm{Gyr}$, and the results are shown in Figure 3. The observed LF is steeper than the SSP model LFs, indicating that the AGB stars in the extraplanar regions of NGC 253 have a range of ages. 


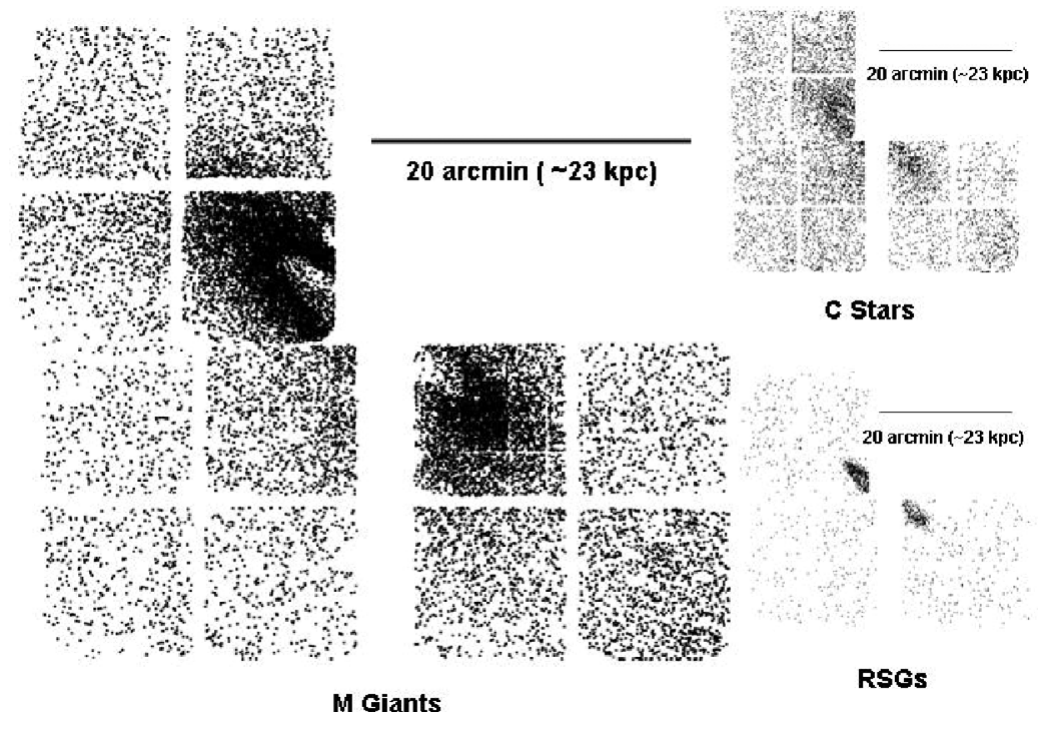

Figure 2. The distribution of M giants, $\mathrm{C}$ stars, and RSGs as selected based on location on the $(K, J-K)$ CMD. North is at the top, and East is to the left. Note the lopsided distribution of M giants, and the diffuse distribution of $\mathrm{M}$ giants in the region around the disk, with sub-structure close to the disk plane. These results are suggestive of an interaction (or interactions) with another galaxy (or galaxies) during intermediate epochs.

The constant SFR model predicts decreasing star numbers towards more luminous $\mathrm{M}_{K}$, and the slope of the model LF is roughly consistent with that of the observed LF. While no effort has been made to finesse the star-forming history to match the observations. Still, it is clear that the LF of extraplanar stars is consistent with them not being part of a coeval system; rather, the extraplanar regions of NGC 253 are populated by stars that formed over a protracted period of time.

\section{DISCUSSION \& SUMMARY}

This study has found evidence that NGC 253 has interacted with another object. There is sub-structure close to the disk plane, and the distribution of RSGs and M giants in the NGC 253 disk is lopsided. Zaritsky \& Rix (1997) find that lopsided galaxies tend to have higher SFRs than symmetric galaxies, and attribute the morphological asymmetry to the accretion of a companion. Finally, the star-forming history of the explanar component suggests that it originated in the disk of NGC 253, and that these stars were disrupted out of the disk plane during the encounter. The distribution on the sky of the extraplanar component is flattened, with an ellipticity near 0.4.

While the orbital properties of the supposed interacting galaxy are not known, there are clues to possible trajectories. The distribution of stars in Figure 2 is suggestive of an encounter involving a companion with an orbit that was close to the NGC 253 disk plane. Simulations run by Hopkins et al. (2009) suggest that the most pronounced asymmetries in the disk result from prograde encounters in which the companion orbit is close to that of the disk plane of the larger galaxy. The lop-sided nature of the NGC 253 disk thus suggests that the galaxy that interacted with NGC 253 was probably not on a retrograde orbit. 

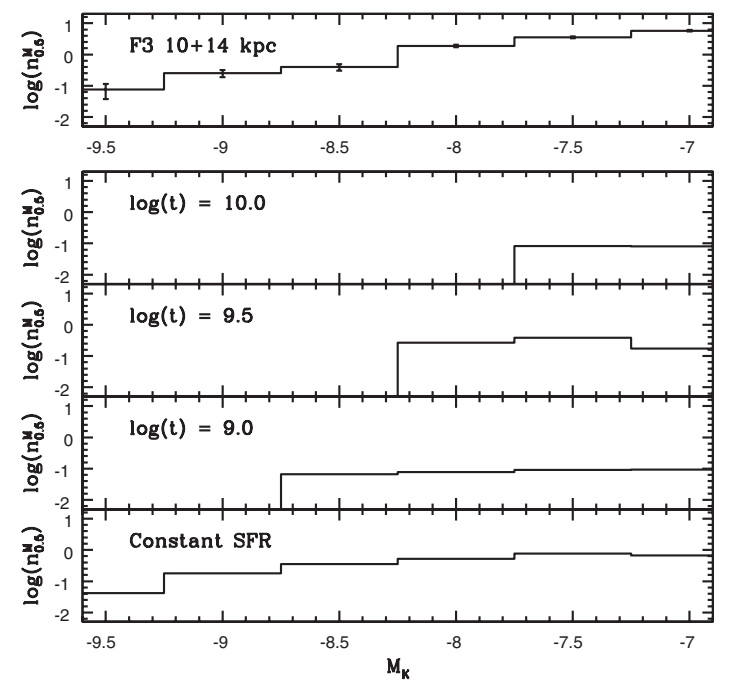

Figure 3. The composite $K$ LF of stars with $J-K$ between 0.8 and 1.6 located between 10 and $14 \mathrm{kpc}$ off of the disk plane is compared with model LFs constructed from the $\mathrm{Z}=0.019$ isochrones of Girardi et al. (2002). $\mathrm{n}_{0.5}^{M}$ is the observed number of stars per square arcmin per 0.5 magnitude interval. The models have been scaled to fall near the center of each panel for display purposes. The comparatively steep downward slope of the observed LF at the bright end indicates that Field 3 contains stars that are not part of an SSP. The panel labelled 'Constant SFR' shows a model LF for a solar metallicity system that has experienced a constant SFR over the time interval 0.2 - 10.0 Gyr. The continuous SFR model better matches the downward trend of the observed LFs towards brighter $\mathrm{M}_{K}$ than the SSP LFs. These comparisons suggest that the intermediate age extraplanar stars in NGC 253 originated in a system that had an active star forming history over Gyr timescales.

\section{References}

Beck, R., Hutschenreiter, G., \& Wielebinski, R. 1982, A\&A, 106, 112

Girardi, L., Bertelli, G., Bressan, A., Chiosi, C., Groenewegen, M. A. T., Marigo, P., Salasnich, B., \& Weiss, A. 2002, A\& A, 391, 195

Harris, J. \& Zaritsky, D. 2001, ApJS, 136, 25

Hoopes, C. G., et al. 2005, ApJ, 619, L99

Hopkins, P. F., Cox, T. J., Younger, J. D., \& Hernquist, L. 2009, ApJ, 691, 1168

Karachentsev, I. D., et al. 2003, A\&SA, 404, 93

Malin, D. \& Hadley, B. 1997, PASA, 14, 52

Zaritsky, D. \& Rix, H-W 1997, ApJ, 477, 118 